\title{
Consumption of Lamb Meat or Basa Fish Shapes the Gut Microbiota and Aggravates Pulmonary Inflammation in Asthmatic Mice
}

This article was published in the following Dove Press journal:

Journal of Asthma and Allergy

\author{
Hao-cheng Zheng $\mathbb{1 D}^{1, *}$ \\ Yong-an Wang ${ }^{1} *$ \\ Zi-rui Liu' \\ Ya-lan Li' \\ Jing-wei Kong' \\ Dong-yu Ge $\mathrm{G}^{2}$ \\ Gui-ying Peng (D) \\ 'Department of Immunology and \\ Microbiology, School of Life Sciences, \\ Beijing University of Chinese Medicine, \\ Beijing I00029, People's Republic of \\ China; ${ }^{2}$ Experimental Teaching Center, \\ School of Traditional Chinese Medicine, \\ Beijing University of Chinese Medicine, \\ Beijing 100029, People's Republic of \\ China
}

*These authors contributed equally to this work
Correspondence: Gui-ying Peng Beijing University of Chinese Medicine, No. II, East Road North Ring 3rd, ChaoYang District, Beijing 100029, People's

Republic of China

Tel +86-010-53912169

Email penggy@bucm.edu.cn
Objective: In China, lamb and fish are well-known triggers for an asthma attack. Our investigation aims at assessing whether the long-term intake of lamb meat or Basa fish would aggravate pulmonary inflammation as well as exploring changes in the intestinal microbiota and immune cells in asthmatic mice.

Materials and Methods: The murine asthmatic model was established by intraperitoneal injection of ovalbumin (OVA) plus aluminum on day 0 and 14 and nebulization of OVA from day 21 to 27 . Lamb meat or fish was administered to asthmatic mice by oral gavage from day 0 to 27 .

Results: Our results showed that long-term consumption of lamb meat or Basa fish in asthmatic mice increased the number of inflammatory cells in bronchoalveolar lavage fluid (BALF), enhanced levels of IL-5, IL-13 in BALF and total IgE in serum, aggravated pulmonary inflammatory cell infiltration and mucus secretion. Long-term oral lamb enhanced the proportion of type 2 innate lymphoid cells (ILC2) from small intestine while it inhibited that of Treg from lung in asthmatic mice. Oral fish showed no remarkable effect on that of ILC2 from lung and small intestine but inhibited that of intestinal Treg in asthmatic mice. What's more, the chao- 1 and observed species richness as well as PD whole tree diversity increased in asthmatic mice while these increments were inhibited after lamb treatment. PCA analysis indicated that there were significant differences in the bacterial community composition after lamb or fish treatment in asthmatic mice. Both lamb and fish treatment enhanced the abundance of colonic Alistipes in asthmatic mice.

Conclusion: Collectively, long-term intake of lamb or fish shapes colonic bacterial communities and aggravates pulmonary inflammation in asthmatic mice, which provides reasonable food guidance for asthmatic patients.

Keywords: asthma, intestinal microbiota, lamb, fish, ILC2, Treg

\section{Introduction}

The prevalence of asthma has increased significantly over the decades. It affects more than 340 million people worldwide and causes at least 250,000 deaths. It creates a massive burden on public health, which leads to a reduced quantity of life and global economic costs. Finding new evidence-based asthma therapies is an effective way to reduce the burden in both developed and developing countries. ${ }^{1,2}$

Diet plays an essential role in many diseases, and it has been described that some kinds of food might have benefits to several diseases such as hypertension, diabetes, and digestive disorders. ${ }^{3}$ However, the association between foods and 
allergic diseases is very complicated. On the one hand, some food, such as vegetable and fruit, has a good effect on asthma. On the other hand, food is also a source of allergies for some people. ${ }^{4}$ Studies have found that many ingredients in food such as n-6 polyunsaturated fatty acid (PUFA) are factors that cause asthma to worsen, and may also be the culprit of provoking asthma. ${ }^{5}$ In traditional Chinese medicine, particular trigger foods, including lamb, fish, onion, and spicy, are believed to aggravate inflammatory diseases, especially allergy. According to Huang's research, asthma is a kind of disease that can be induced seriously by taking trigger food, while lamb and fish meat are these two typical foods. ${ }^{6} \mathrm{Zhu}$ found that intake of lamb could increase the levels of TNF- $\alpha$, IFN$\gamma$, and IL-17 in the serum of dermatitis mice. Fish is also found not conducive to wound healing and instead exacerbates inflammation in the traumatic model. ${ }^{7}$ Additionally, a food allergy may be associated with allergic asthma. ${ }^{8}$ Nevertheless, there are still few studies on the relationship between trigger foods and asthma.

In recent years, more and more researchers focus on the nutrition-gut microbiome-physiology axis. Changing diet patterns to shape the gut microbiota has become a potential treatment to alleviate diseases. ${ }^{9}$ The pork can induce oxidative stress and inflammation by altering gut microbiota while the effect and mechanism of lamb and fish on microbes is still not thoroughly explained. ${ }_{-}^{10-12}$ Intestinal microbiota shows a strong relationship with susceptibility and severity of asthma. ${ }^{13}$ Studies have shown that the early reduction of diversity in the intestinal microbiota is closely related to the child's asthma phenotype. ${ }^{14}$ Intestinal clearance of mice by antibiotics can aggravate allergic airway inflammation induced by airway allergens. ${ }^{15}$ Besides, some probiotics such as Bifidobacteria and Lactobacilli have been tried in the treatment of asthma. The study finds Bifidobacteria relieves asthma by regulating Th1/Th2 balance, and supplementation with Lactobacillus rhamnosus relieves airway inflammation of asthmatic mice. ${ }^{16-18}$ Therefore, how nutrition shapes intestinal microbiota is crucial to understand the mechanism of the development of asthma.

Treg and ILC2 cells play essential roles in the occurrence of allergic diseases. Treg can downregulate the immune response to alleviate inflammation. Therefore, the decrease of Treg may be one of the pathological mechanisms of asthma. ${ }^{19,20}$ Recently ILC2 has been proved to contribute to the development of asthma. Although it only accounts for a small part of the immune cells in the lungs under normal conditions, it is the primary producer of IL-5 and IL-13 in the lung. ${ }^{21,22}$ ILC2 can be divided into two populations, including the inflammatory ILC2, which is marked as $\mathrm{KLRG}^{+}{ }^{+} \mathrm{ILC} 2$, and natural ILC2 as $\mathrm{ST}_{2}{ }^{+} \mathrm{ILC}_{2}{ }^{23}$ Both $\mathrm{KLRG}^{+}{ }^{+}$ILC2 2 and Treg have tissue migration function. ${ }^{24-27}$ Thus, our study aims at exploring whether two trigger foods, including lamb and fish, will aggravate pulmonary inflammation as well as how gut microbiota and immune cells will be shaped in OVA-induced asthmatic mice.

\section{Materials and Methods}

\section{Animals}

The study was conducted at the Beijing University of Chinese Medicine. Female BALB/c mice, 6-8 weeks old, were purchased from Beijing Vital River Laboratory Animal Technology Company (Beijing, China). Animals were fed in the SPF animal room of Beijing University of Chinese Medicine. All animal studies were approved by the Animal Ethics Committee of Beijing University of Chinese Medicine (Approval Number: BUCM4-2018120601-4054). Animal experiments were conducted according to the guidelines of the Animal Care and Use Committee of Beijing University of Chinese Medicine.

\section{Preparation of Food Mixture and Application}

The raw materials are purchased from Jindong Online Mall (Jingdong.com). Here lamb meat (The following abbreviation is lamb) and Basa fish (The following abbreviation is fish) are chosen as the raw materials. The HU's methods were adopted and modified as followed. ${ }^{28} 500 \mathrm{~g}$ of lamb meat or Basa fish was poaching and boiling for 3 hours after being washed. Then the boiling was homogenized, and diluted with sterilized water to a final volume of $1000 \mathrm{~mL}$ for the stomach filling. $0.2 \mathrm{~mL}$ of food mixture at a concentration of $0.5 \mathrm{~g} / \mathrm{mL}$ was given daily per mouse from day 0 to 27 , according to $40 \mathrm{~g}$ daily intake of lamb or fish for humans.

\section{Establishing Allergic Asthma}

The allergic asthmatic murine model was established and modified according to methods of $\mathrm{Xu}^{29}$ After one week of adaptation to the environment, $\mathrm{BALB} / \mathrm{c}$ mice were randomly divided into four groups with at least six mice each, as follows: (1) PBS group (2) OVA group (3) OVA+Lamb 
group (4) OVA+Fish group. On day 0 and 14, the mice in the OVA, OVA+Lamb, and OVA+Fish groups were sensitized by intraperitoneal injection with $20 \mu \mathrm{g}$ of OVA (Sigma-Aldrich $^{\mathrm{TM}}$, St. Louis, MO, USA) in PBS mixed with an equal volume of Alum as an adjuvant in a total volume of $200 \mu \mathrm{L}$. On day 21-27, these mice were nebulized in an atomization chamber (1\% OVA in PBS) for 30 mins per day. For the PBS group, PBS is applied to replace OVA instead. The body weights of the mice were recorded daily during the experimental process.

\section{Serum, Bronchoalveolar Fluid Collection and Cell Counting}

On day 28, mice were anesthetized with isoflurane and euthanized by cervical dislocation after collecting blood. Serum was obtained by centrifuging the blood at $4500 \mathrm{~g}$ for 15 minutes at $4{ }^{\circ} \mathrm{C}$ and stored at $-80^{\circ} \mathrm{C}$ until analysis. The lungs of the mice were lavaged three times, with $1 \mathrm{~mL}$ of normal saline containing 1\% FBS (FBS, Gibco). BALF was centrifuged $(1500 \mathrm{~g})$ for 5 minutes at $4^{\circ} \mathrm{C}$, and the supernatant was stored at $-80^{\circ} \mathrm{C}$ for detecting cytokines. The precipitation was suspended by PBS, diluted 1000 times and stained with trypan blue. Finally, the cell numbers of BALF were counted under the microscope.

\section{Histological Examination of the Lungs}

Histological examination is used to assess disease progression further. The lungs were washed in phosphate buffer and fixed in $10 \%$ formaldehyde at room temperature. The organs were dehydrated in a gradient of ethanol and then embedded in paraffin. Tissue sections of $4 \mu \mathrm{m}$ were stained with hematoxylin and eosin (H\&E) and periodic acid-Schiff (PAS) to assess pathological morphology.

\section{Enzyme-Linked Immunosorbent Assay (ELISA)}

Total IgE levels in serum (Biolegend, 432401), and IL-5 (Biolegend, 431204), IL-13 (eBioscience, KMC2221) levels in BALF were measured by enzyme-linked immunosorbent assay (ELISA) kit according to the manufacturer's instructions.

\section{Cell Isolation from Lung and Gut Tissues in Mice}

A single-cell suspension was obtained according to the reference. ${ }^{30}$ In brief, mice were anesthetized with isoflurane and euthanized by cervical dislocation on day 28 .
Lungs were minced and incubated with $1 \mathrm{mg} / \mathrm{mL}$ collagenase IV (Worthington ${ }^{\mathrm{TM}}$, LS004189) and $50 \mu \mathrm{g} / \mathrm{mL}$ DNase I (Roche ${ }^{\mathrm{TM}}$, 11284932001) in RPMI-1640 media for 40 min at $37^{\circ} \mathrm{C}$ before being mashed through $70 \mu \mathrm{m}$ cell strainers. For isolation of cells from the intestine, after adherent fat tissue and Peyer's patches were removed, the gut was opened longitudinally, cut into $5 \mathrm{~mm}$ pieces, and washed with $20 \mathrm{~mL}$ HBSS medium containing $5 \mathrm{mM}$ EDTA and $1 \mathrm{mM}$ DTT for $20 \mathrm{~min}$ at $37^{\circ} \mathrm{C}$ twice to remove epithelial cells. Then, gut tissues were digested with $2 \mathrm{mg} /$ $\mathrm{mL}$ collagenase type III (Worthington, LS004183) and 50 $\mu \mathrm{g} / \mathrm{mL}$ DNase I in RPMI-1640 media for $40 \mathrm{~min}$. Singlecell suspensions from all tissues were used for subsequent flow cytometry staining.

\section{Flow Cytometry Analysis}

Single-cell suspensions were kept at $4^{\circ} \mathrm{C}$, and nonspecific binding was blocked by anti-Fc receptor blocking antibody (anti-CD16/32) before cell surface staining. For detecting ILC2, cells were first stained with biotinylated monoclonal antibodies specific for CD3e, CD4, CD8 $\alpha$, CD11c, CD11b, CD19, TCR $\beta$, TCR $\gamma \delta$, NK1.1, Gr1, Ter119, and B220. Then these cells were stained with Alexa Fluor ${ }^{\circledR} 488$ conjugated streptavidin, APC-Cy7 conjugated anti-mouse CD45, PE-Cy7 conjugated anti-mouse CD90.2, Percp-5.5 conjugated anti-mouse KLRG1.For Treg detection, cells were first stained with FITC anti-mouse CD3, PE antimouse CD4, and APC anti-mouse CD25. Then these cells were stained with Percp-5.5 anti-mouse FOXP3 after being fixed and permed with intracellular Fixation \& Permeabilization Buffer (eBiosciences, 88882400). Cells were detected by FACS CantoTM (BD, Biosciences) and analyzed by FlowJo software.

\section{I6S rDNA Gene Microbiome Analysis}

DNA samples taken from BALB/c mouse feces in different groups were analyzed based on $16 \mathrm{~S}$ rDNA gene sequences to examine characteristic bacterial communities along the intestinal tract of mice. Small fragment libraries with concentrations higher than $30 \mathrm{ng} / \mu \mathrm{L}$ were used for PCR amplification. After thawing on ice, the samples were tested before treatment, centrifuged and thoroughly mixed, and the Qubit test was used to test the sample concentration. The PCR program conditions were set at $95^{\circ} \mathrm{C}$ for 5 minutes, then $95^{\circ} \mathrm{C}$ for 30 seconds, then $56^{\circ} \mathrm{C}$ for 30 seconds, 25 cycles, then $72^{\circ} \mathrm{C}$ for 40 seconds, then $72^{\circ}$ $\mathrm{C}$ for 10 minutes, and finally at $4^{\circ} \mathrm{C}$. Detected on a $2 \%$ $(150 \mathrm{~V}, 30 \mathrm{mins})$ agarose gel. The universal primers were 
$\left(5^{\prime}\right.$-ACTCCTACGGGAGGCAGCA-3') and $\left(5^{\prime}\right.$ GGACTACHVGGGTWTCTAAT-3'), based on $16 \mathrm{~S}$ rDNA sequencing, using the two hypervariable regions of the 16S rRNA, V3 and V4 regions to identify the majority of bacteria, and the positive and negative readings of the V3 and V4 regions of the Illumina HiSeq 2000 platform. Only sequences longer than $50 \mathrm{bp}$ were used for systematic analysis at the 0.03 Operational Taxonomic Units (OTU) level. All procedures were performed following the manufacturer's agreement (Allwegene Company, Beijing, China). Bioinformatics analysis will be performed along with the sequencing data.

\section{Statistical Analysis}

All data were expressed as mean \pm SEM. Data analysis was performed using SPSS20.0 software. Data comparison between the two groups was performed by Student's $t$-test. Data comparison between more than two groups was analyzed by one-way ANOVA. $P<0.05$ are considered statistically significant.

\section{Results}

\section{Changes in the General State of the Mice} In our study, an asthmatic model was established by OVA sensitization and challenge (Figure 1A) Lamb and fish was administrated by intragastric injection to asthmatic mice from day 0 to 27 . Considering the effect of foods on body weight, the body weights of all mice throughout the whole experiment were detected. Overall, OVA, OVA+Lamb, and OVA+Fish groups indicated a significant weight loss compared with the PBS group on $\mathrm{d} 27$ (Figure 1B). Among them, that from the OVA +Lamb group showed the most obvious. Although the comparison of the length of the small intestine of the four groups showed no significant difference, the length of the colon from the OVA+Lamb group was significantly shortened compared with the OVA group $(P<0.05)$. It indicated that lamb meat probably contributed to colonic inflammation in asthmatic mice. What's more, the lung and spleen indices of the OVA group, the OVA+Lamb group, and the OVA+Fish group were considerably higher than those of the PBS group. Compared with the OVA group, the spleen index of the OVA + Lamb group rose significantly $(P<0.05)$ (Figure $1 \mathrm{C}$ ).

\section{Lamb and Fish Aggravates Pulmonary Inflammation in Asthmatic Mice}

In the OVA-induced asthma mouse model, increasing serum total $\mathrm{IgE}$ and inflammatory cells, infiltration in BALF are the most characteristic of asthma. Compared

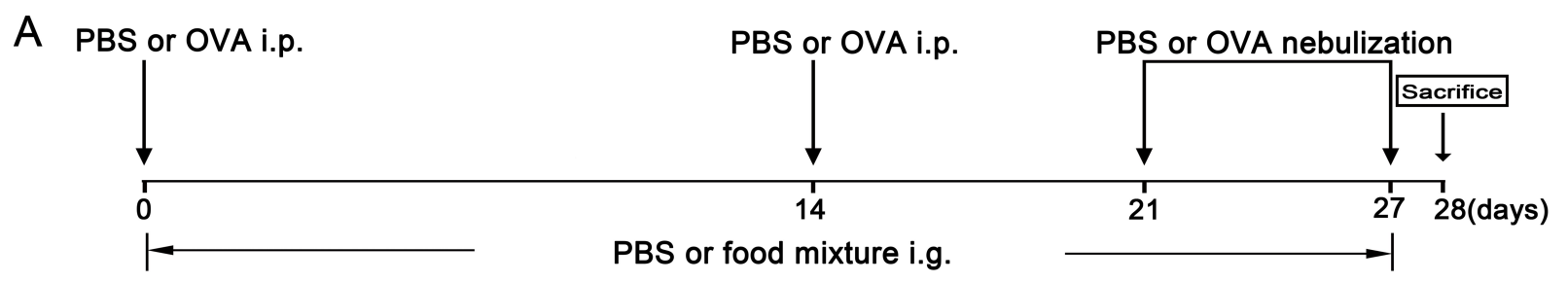

B

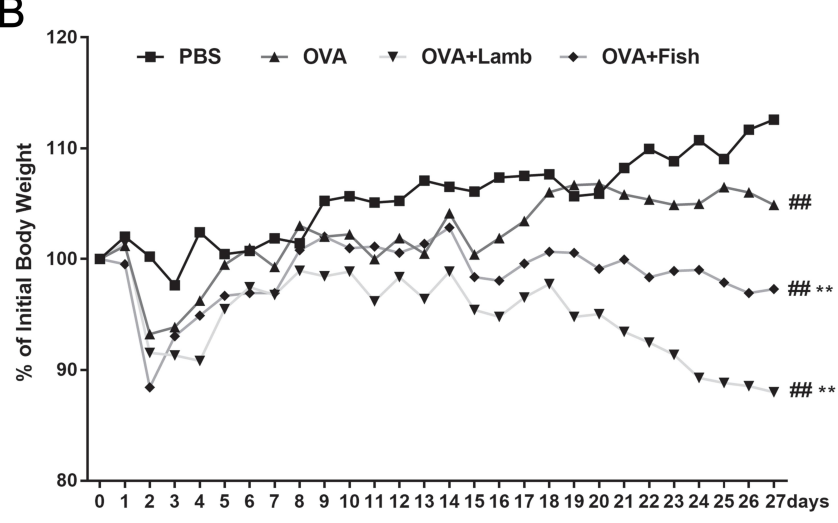

C
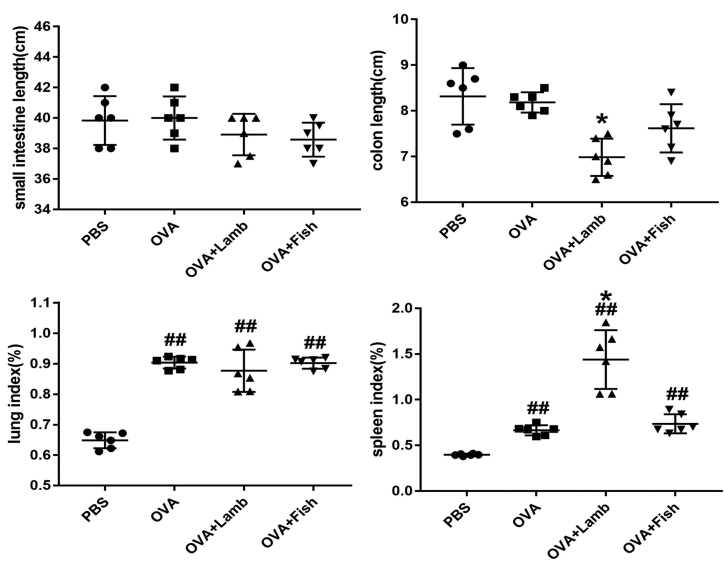

Figure I Effect of lamb and fish on the body weight, gut length, lung and spleen index of mice. (A) A schematic view for the induction of allergic asthma along with lamb or fish treatment in mice. (B) The body weight change curve of mice. The weights among different groups were compared on d27. (C) On d28, large intestine, small intestine, lung, and spleen are removed. Gut length, lung and spleen index was calculated. Lung index = lung weight $(\mathrm{g}) / \mathrm{body}$ weight $(\mathrm{g}) \times 100 \%$; Spleen index $=$ spleen weight $(\mathrm{g}) / \mathrm{body}$ weight $(\mathrm{g}) \times 100 \%$. All data were expressed as mean \pm SEM. Compared with the PBS group, ${ }^{\#} \mathrm{P}<0.01$; compared with the OVA group, $* \mathrm{P}<0.05$. $\mathrm{n}=6 \mathrm{per}$ group. 
with the PBS group, the OVA group developed severe peripheral vascular and bronchial inflammation, which manifested as infiltration of leukocytes around the bronchi and secretion of mucus, as well as thickening of the alveolar wall and narrowing of the alveolar space (Figure 2A). Compared with the OVA group, these damages of the OVA+Lamb and OVA+Fish group were more evident with a significantly increasing number of goblet cells by PAS staining and remarkable leukocyte infiltration in HE staining, especially in OVA+Lamb group. A significant increase in IgE concentration was observed in the OVA group $(P<0.01)$, while the OVA+Lamb and OVA+Fish group showed a higher IgE concentration $(P<0.01$ of OVA+Lamb group, $P<0.01$ of the OVA+Fish group). Cells from BALF were collected at the end of the experiment. The total numbers of inflammatory cells in the OVA +Lamb and OVA+Fish group were both increased significantly compared to the OVA group $(P<0.01$ of OVA + Lamb group, $P<0.05$ of the OVA+Fish group). Furthermore, the concentration of IL-5 and IL-13 in BALF was significantly increased in the OVA+Lamb and OVA+Fish group compared with the OVA group, which is consistent with mucus release in pathological sections (Figure 2B).

\section{Lamb and Fish Changes Gut Microbial Community Structure in OVA-Induced Asthmatic Mice}

16SrDNA analysis was performed to investigate further how lamb and fish shape intestinal flora in asthmatic mice. In total, 941,480 valid sequences were measured, with an average of 78,456 sequences per sample. The analysis was performed at a level of similarity higher than $97 \%$, and a total of 1076 OTUs were obtained. The PBS group has an average of 343 OTUs, OVA group 593, OVA+Lamb 395, and OVA+Fish 559 (Table 1). The curves of all samples tend to be flat, indicating that the amount of sequencing data is large enough to reflect most microbial information in the sample.

Next, samples from four groups were compared regarding the abundance and diversity of microbiota. The results showed that the abundance and diversity of the OVA group increased significantly compared with the PBS group $(P<0.01)$. Compared with the OVA group, the abundance and diversity of the OVA+Fish group did not change much. Although the diversity of the OVA+Lamb group did not change significantly either, it is worth noting that Chao index and observed_species of the OVA+Lamb group showed a significant decrease compared with the
A
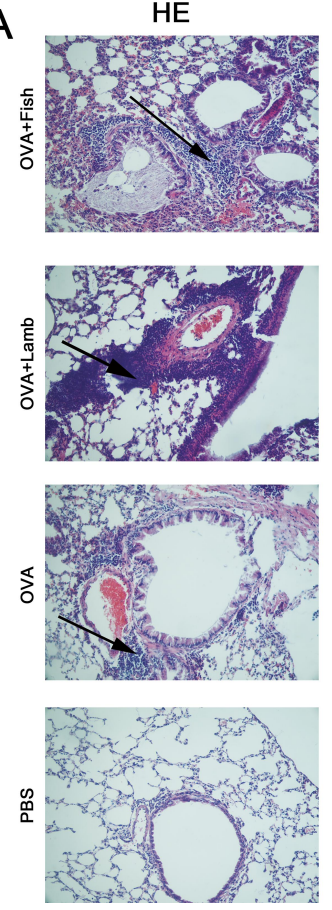

PAS
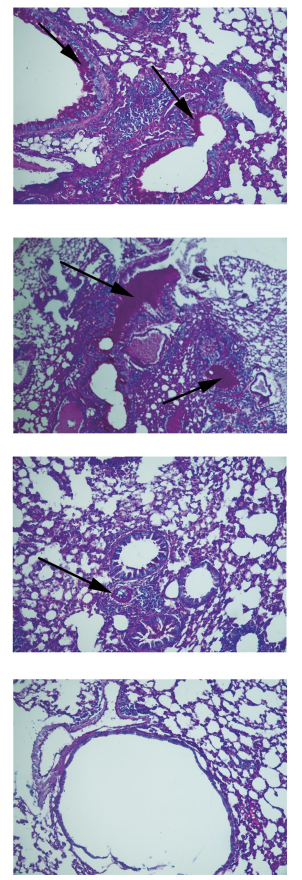

B
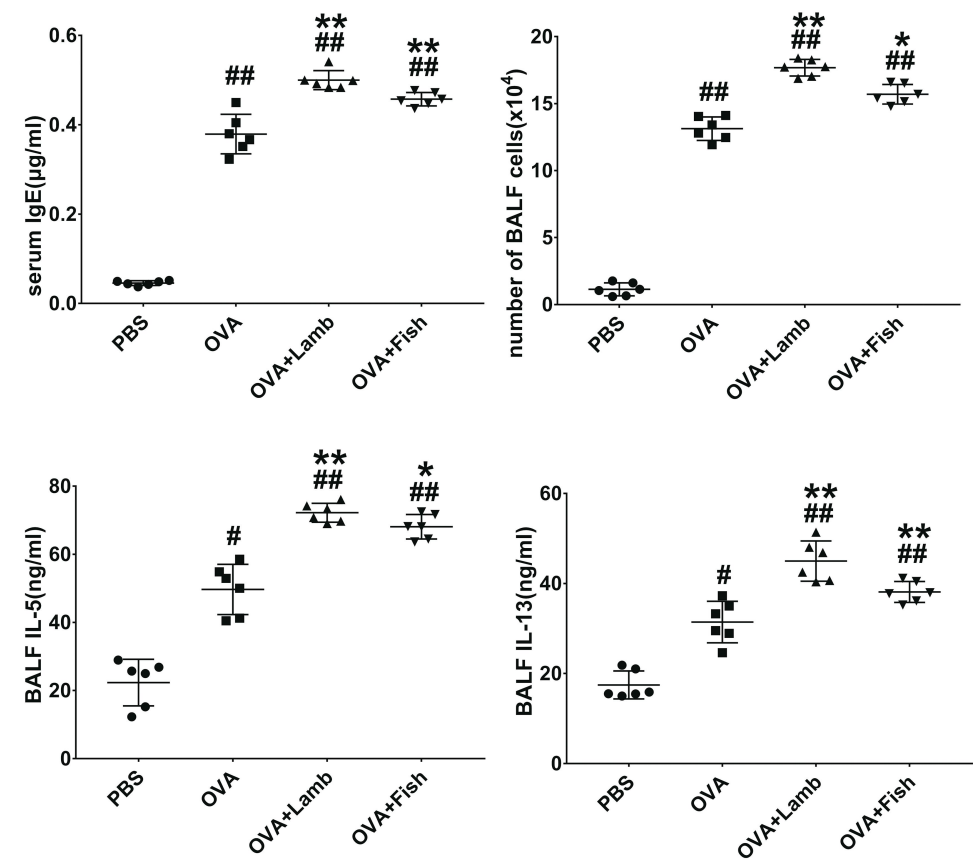

Figure 2 Effect of lamb and fish on the lung inflammation of mice. (A) Lung histological effects of lamb and fish in asthmatic mice (magnification $\times 20$ ); (B) IgE levels from serum, the total cell numbers from BALF, IL-5, and IL-I3 levels from BALF. All data were expressed as mean \pm SEM. Compared with the PBS group, ${ }^{\#} P<0.0$ I ${ }^{\#} P<0.05$; compared with the OVA group, $* * P<0.01 * P<0.05$. $n=6$ per group. 
Table I Comparison of Bacterial Communities' Diversity in the Murine Colon Among Four Groups ${ }^{\mathrm{a}}$

\begin{tabular}{|l|l|l|l|l|}
\hline \multirow{2}{*}{ Sample ID } & \multirow{2}{*}{$\begin{array}{l}\text { Valid } \\
\text { Sequences }\end{array}$} & \multicolumn{3}{|c|}{ Similarity Score $\geq \mathbf{0 . 9 7}$} \\
\cline { 3 - 5 } & & OTU & Chao-I & Shannon \\
\hline PBS I & 28,399 & 327 & 408.42 & 4.46 \\
PBS 2 & 42,710 & 373 & 465.63 & 4.76 \\
PBS 3 & 22,337 & 329 & 454.28 & 4.62 \\
OVA I & 80,606 & 594 & 722.33 & 6.41 \\
OVA 2 & 97,557 & 589 & 750.62 & 6.03 \\
OVA 3 & 79,638 & 597 & 778.06 & 6.47 \\
OVA+Lamb I & 40,772 & 393 & 594.81 & 6.50 \\
OVA+Lamb 2 & 78,561 & 356 & 476.07 & 5.95 \\
OVA+Lamb 3 & 105,402 & 437 & 433.17 & 5.92 \\
OVA+Fish I & 178,645 & 478 & 630.94 & 6.69 \\
OVA+Fish 2 & 70,255 & 570 & 745.20 & 6.30 \\
OVA+Fish 3 & 116,598 & 630 & 844.93 & 6.36 \\
\hline
\end{tabular}

Note: ${ }^{a}$ Sequences with similarity scores greater than or equal to 0.97 were clustered into an OTU.

OVA group, suggesting a significant reduction in the abundance of gut microbes $(P<0.01)$ (Figure 3A). PCA analysis found that the differences among the four groups were significant. The more similar the composition of the microbiota between samples, the closer the distance representing their coordinate points in the PCA plot. The contribution rates of $\mathrm{PC} 1$ and $\mathrm{PC} 2$ in Figure 3B were 40.92 and $24.26 \%$ respectively, with a cumulative contribution rate of $65.18 \%$. The closer the PCA plot in the distribution principal components in Figure 3B, the more similar the community composition of the samples. On the PC2 axis,
PBS and OVA+Lamb treatments were distributed towards the positive direction, while OVA and OVA+Fish treatments were mainly distributed towards the negative direction. The PBS group was significantly different from the other three groups, and the OVA+Lamb group was significantly diverse from the OVA group or OVA+Fish group (Figure 3B).

\section{Lamb and Fish Changes the Taxa Abundance of the Intestinal Flora in OVA-Induced Asthmatic Mice}

To explore potential probabilities of the observed changes in diversity, we continued to analyze the relative abundance of intestinal flora. At the phylum level, we found that Firmicutes and Bacteroides accounted for more than $95 \%$ of the total gut flora. There was no significant difference among the PBS group, the OVA group, and the OVA + Fish group (Figure 4A). However, compared with the OVA group, Firmicutes from the OVA+Lamb group increased significantly $(P<0.01)$ while Bacteroidetes decreased significantly $(P<0.01)$ (Figure 4A).

At the genus level, compared with the PBS group, the relative abundance of Lactobacillus $(P<0.01)$ and Rikenellaceae_RC9_gut_group $(P<0.05)$ from the OVA group, the OVA+Lamb group, and the OVA+Fish group decreased significantly, while the relative abundance of Lachnoclostridium $(P<0.05)$ increased significantly. Compared with the OVA group, the relative abundance
A
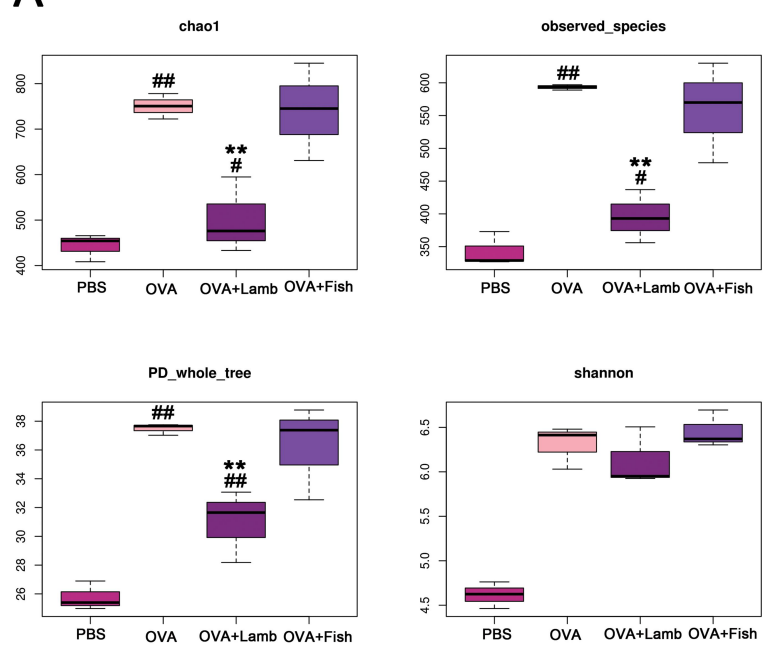

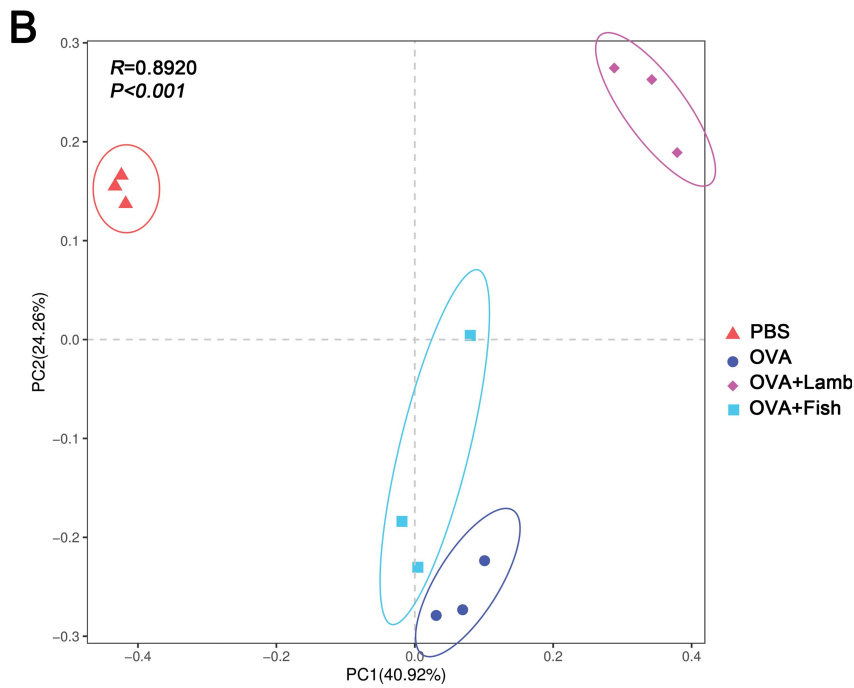

Figure 3 Effect of lamb and fish on the intestinal microbiota of mice. (A) Comparison of alpha diversity of Chao index, observed species, PD whole tree, and Shannon's index among four groups. (B) Principal Component Analysis (PCA) of mice with ANOSIM analysis. Compared with the PBS group, ${ }^{\#} P<0.0$ I ${ }^{\#} P<0.05$; compared with the OVA group, $* * P<0.01 . \mathrm{n}=3$ per group. 


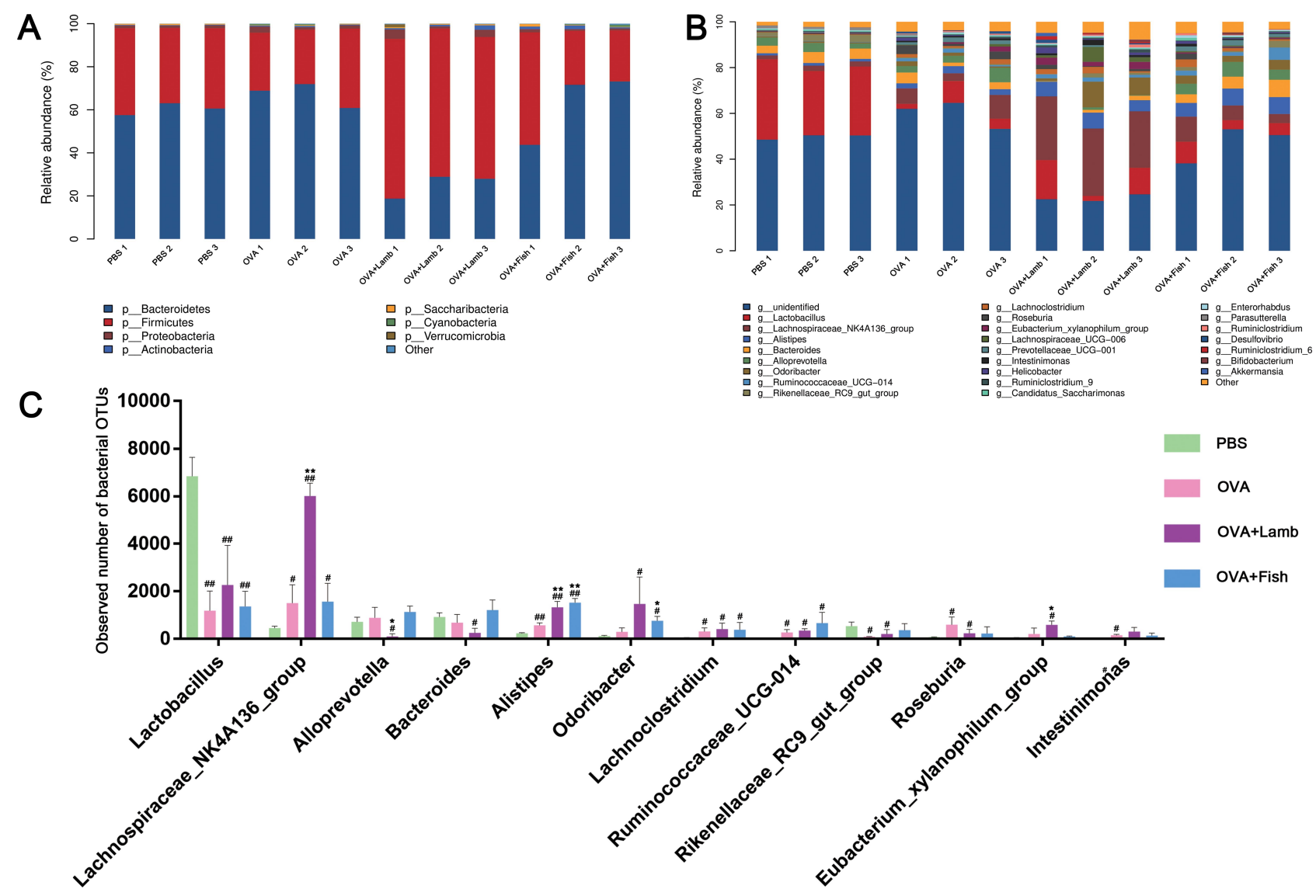

Figure 4 Effect of lamb and fish on the taxa abundance of intestinal flora in mice. (A) The relative abundance of the phylum level. (B) The relative abundance of genus level. (C) Top 12 relative bacterial abundance of four groups at the genus level. All data were expressed as mean \pm SEM. Compared with the PBS group ${ }^{\#} P<0.0$ I ${ }^{\#} P<0.05$; compared with the OVA group, $* * P<0.01 * P<0.05$. $\mathrm{n}=3$ per group.

of Lachnospiraceae_NK4A136_group from the OVA + Lamb group increased significantly $(P<0.01)$ while the relative abundance of Alistipes showed remarkable enhancement in both the OVA+Lamb group $(P<0.01)$ and OVA+Fish group $(P<0.01)$ (Figure $4 \mathrm{~B})$. Therefore, the flora in the top twelve relative abundances was listed in the Figure $4 \mathrm{C}$, considering a vast difference in the relative abundance of the genus levels among the four groups.

\section{Analysis of Intestinal Flora LEFSe}

We further used linear discriminant analysis effect size (LEFSe) to explore the crucial flora associated with asthma and lamb or fish treatment. The cladogram in Figure 5A showed the core bacterial species with remarkable differences at all levels. The bacterial members with significantly high abundance mainly belonged to the Bacillaceae in the PBS group. In the OVA group, there were two families with a significantly greater population, Bacteroidales_S24_7_group and Carnobacteriaceae. After Lamb + OVA treatment, the relative abundance of the bacterial taxa with a significantly greater population was Lachnospiraceae, Ruminococcaceae, Clostridiales and Clostridia. There were two families with a significantly greater population, Prevotellaceae and Rikenellaceae in OVA+Fish group.

The distribution histogram (Figure 5B) indicated that three specific taxa existed in the PBS group and influenced the bacterial composition. Two specific taxa were found in the OVA group, Bacteroidales_S247_group and Carnobacteriacear, which influenced the community structural composition. Five specific taxa were found after OVA+Lamb treatment which influenced microbial community structure. In the OVA+Fish group, there were six significant taxa found.

\section{The Effect of Lamb and Fish on ILC2 and Treg in Asthmatic Mice}

Considering the pivotal roles of ILC2 and Tregs in the development of asthma, we analyzed the proportion of ILC2 and Tregs in the lung and small intestine. Gating 


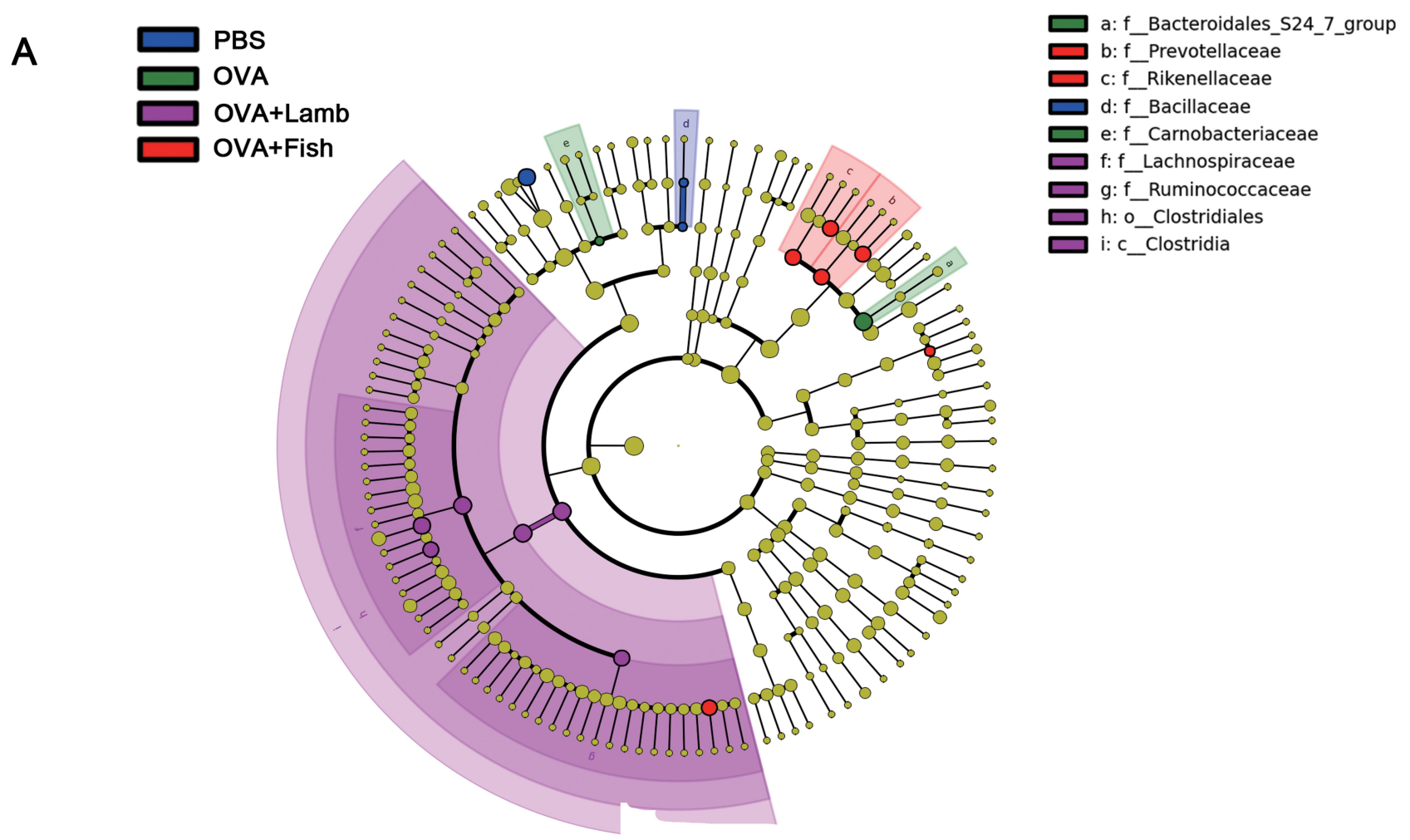

B
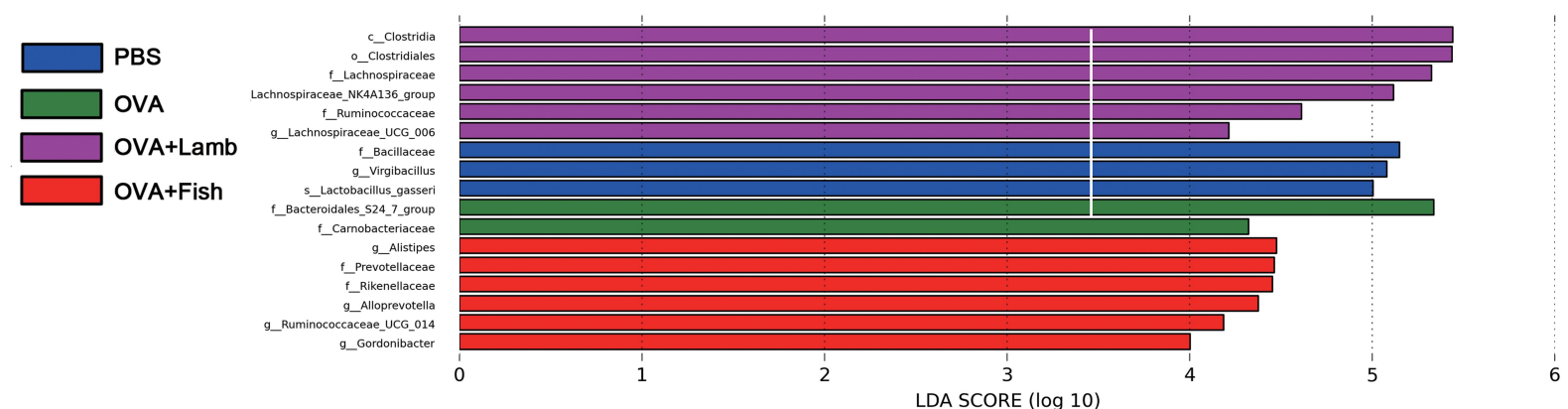

Figure 5 LEFSe analysis of mouse intestinal flora among four groups. (A) Cladogram of each group. (B) LDA values of each group. The LDA significant threshold was 4.0. Blue, green, purple, and red represented PBS, OVA, OVA+Lamb and OVA+Fish, respectively. $n=3$ per group.

strategy for ILC2 and Tregs are shown in Figure 6A and B. Though ILC2 only accounts for a small part of CD45 ${ }^{+}$cells in the lung, the proportion of ILC2 in the OVA group increased, compared with the PBS group $(P<0.05)$. However, these two trigger foods did not change the proportion of lung ILC2 in asthmatic mice. There was no significant difference in the proportion of ILC2 from the small intestine between the PBS group and the OVA group, but there existed a significant increase in OVA + Lamb group $(P<0.05)$ (Figure $6 \mathrm{C})$. These suggested that oral lamb treatment in asthmatic mice probably changed the gut microbial community and induced intestinal ILC2 expansion.

What's more, there existed a significant reduction in the proportion of Treg in the lung from the OVA group compared with the PBS group, while lamb treatment exacerbated this decrease $(P<0.05)$ (Figure 6D). There was no significant difference in intestinal Tregs between the PBS group and the OVA group. However, fish treatment rendered the noticeable lowering in asthmatic mice $(P<0.05)$ (Figure 6D). Considering the immune inhibition of Tregs, these results further approved that aggravation of oral lamb on pulmonary inflammation might be executed by circulating inflammatory cytokines, not by immune cells' trafficking.

\section{Discussion}

Diet is an integral part of treatment and plays a vital role in improving the life quality of patients. Our investigation 

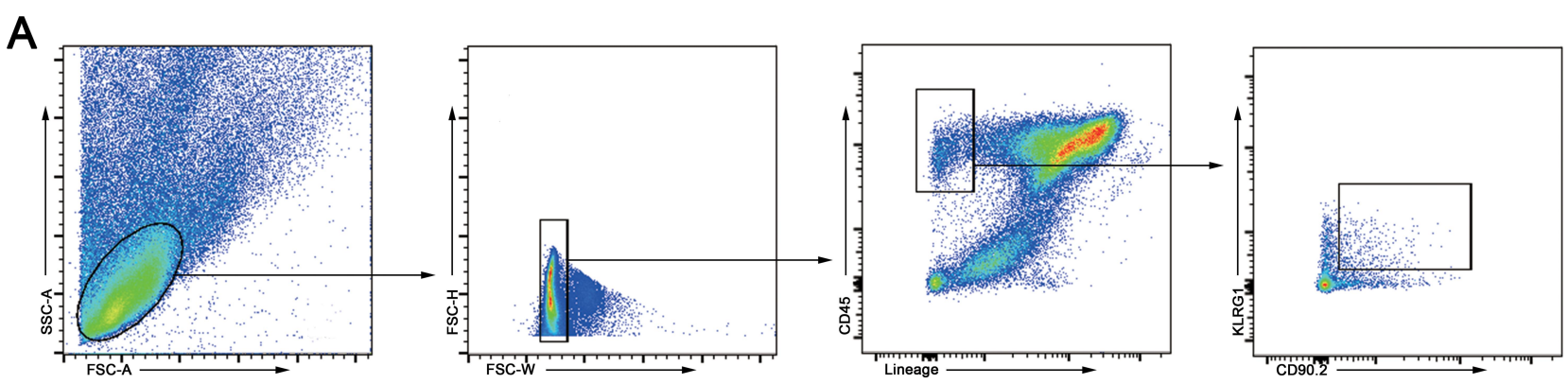

B
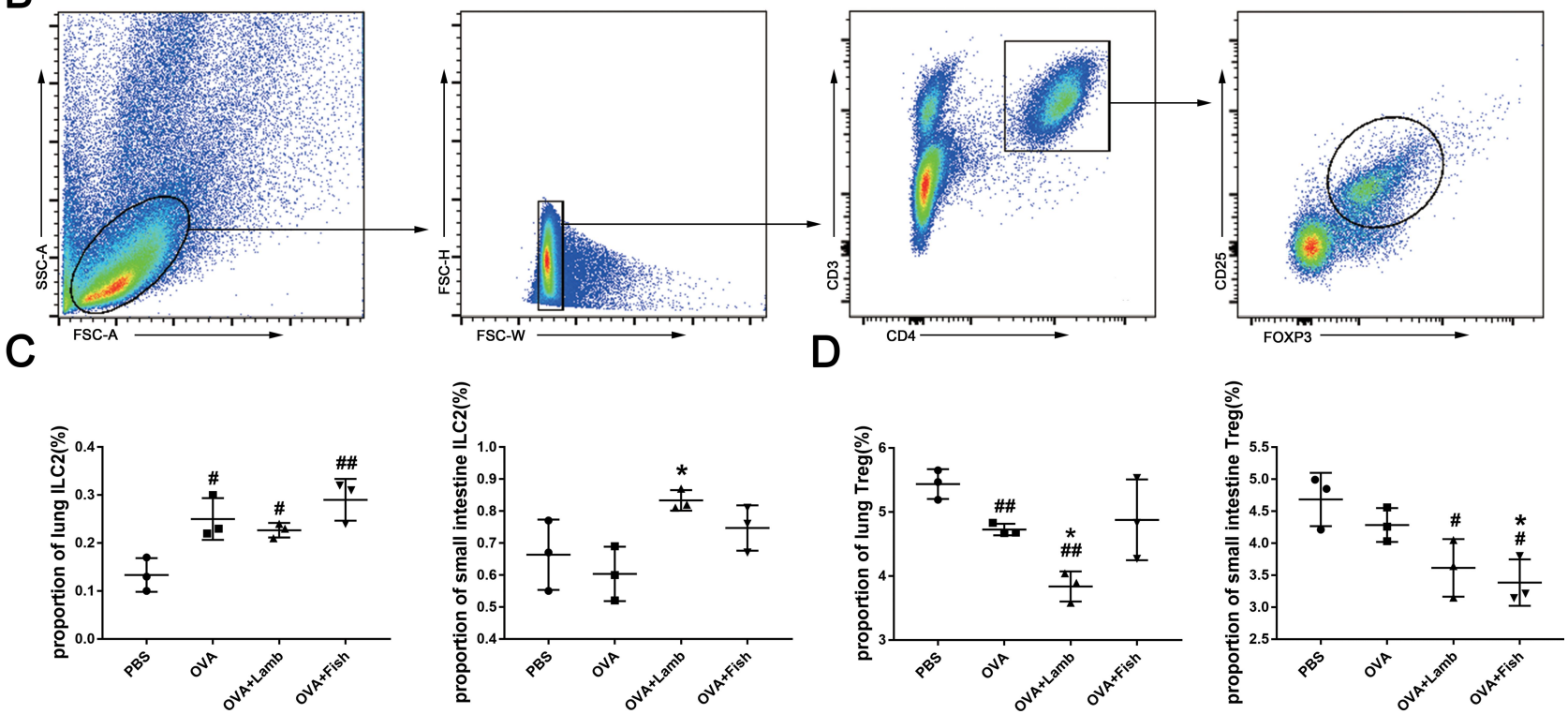

Figure 6 Effect of lamb and fish on ILC2 and Treg. (A) The gating strategy of ILC2. Lin-CD45 ${ }^{+} \mathrm{CD} 90.2^{+} \mathrm{KLRGI}{ }^{+}$cells were considered as ILC2. (B) The gating strategy of Treg. Treg was indicated as $\mathrm{CD} 3^{+} \mathrm{CD} 4^{+} \mathrm{CD} 25^{+} \mathrm{Foxp}^{+}$. (C) The proportions of KLRGI ${ }^{+} \mathrm{ILC} 2$ among $\mathrm{CD} 45^{+}$cells in the lungs and small intestines. (D) The proportions of Treg among $\mathrm{CD}^{+}$cells in the lungs and small intestines. All data were expressed as mean $\pm \mathrm{SEM}$. Compared with the PBS group, ${ }^{\# \#} P<0.01{ }^{\#} \mathrm{P}<0.05$; compared with the OVA group, $* P<0.05 . n=3$.

demonstrates the effects of two typical foods on asthmatic mice. It shows that both lamb meat and Basa fish can aggravate pulmonary inflammation of asthmatic mice, but the impact of lamb on asthma is more apparent. Lamb and fish worsened the pathological inflammatory cell exudation and mucus distribution in asthmatic mice. The rise of IL-5 and IL-13 can also confirm this point.

Andrianasolo found that consumption of highly processed meat was positively correlated with the severity of asthma symptoms. ${ }^{31}$ A Japanese survey of 1673 residents showed that fish intake was positively correlated with asthma risk. ${ }^{32}$ Furthermore, it is shown that the main components of lamb were $20.33 \%$ protein and $12.41 \%$ fat (FoodData Central, FDC ID: 172617 ), and $16.2 \%$ protein and $2.8 \%$ fat for catfish (FoodData Central, FDC ID: 473858). ${ }^{33}$ Fatty acids from lamb meat were mainly oleic acid and palmitic acid, ${ }^{34}$ a prospective study through 420 patients hosted by European
Prospective Investigation into Cancer and Nutrition (EPIC) found that high intake of oleic acid and asthma were positively correlated, ${ }^{35}$ which could be a reason why the effect of lamb on asthmatic mice is more significant than fish.

It is worth noting that some scientists believe that n-3 and n-6 fatty acids, which are rich in fish, have a preventive effect on asthma. ${ }^{36,37}$ But there is no direct evidence of the impact of these fatty acids on asthma. We used the OVA-induced asthmatic model to find that consumption of large doses of Basa fish for 27 days aggravated the inflammation of asthmatic mice. Besides, some researchers have found that 28 days of high-fat diet will increase the LPS content in the blood by $2-3$ times. ${ }^{38}$ And the increase of LPS in the blood can enhance the systemic inflammatory reaction. ${ }^{39}$ Therefore, we believe that there is a specific relationship between fish intake and its effect on asthma. 
The study of the interaction between asthma and intestinal flora is a trend that has attracted increasing attention in asthma research, including curing asthma through taking probiotics such as Bifidobacteria and Lactobacilli. ${ }^{40,41}$ It was found that the consumption of lamb had a more considerable influence on the intestinal flora than that of fish in asthmatic mice. Chao-1 and Shannon index were essential indicators of abundance and diversity of gut microbiota, respectively. The Chao-1 index in the OVA+Lamb group changes significantly compared with the OVA group, while the change of Shannon was not noticeable. It indicates that the abundance of particular flora in the OVA+Lamb group changed significantly, which could be the mechanism of lambs to aggravate inflammation. Therefore, we further analyzed the relative abundance of intestinal flora and found that Alistipes in OVA+Lamb and OVA+Fish groups increased significantly. Alistipes is the dominant richness bacteria in IL-10 mediated enteritis, which can lead to an imbalance of the intestinal state. ${ }^{42}$ Meanwhile, it is found that Lactobacillus in OVA, OVA+ Lamb, and OVA+ Fish groups are all decreased, which is consistent with the reports of the treatment effect of Lactobacillus. ${ }^{18}$ And researchers found that Lactobacillus feeding can lead to a decrease in Alistipes abundance. ${ }^{43}$ This indicates that Lactobacillus may have a mechanism of inhibiting Alistipes, and the reduction of Lactobacillus will lead to the increase of Alistipes abundance, which is consistent with the decrease of Lactobacillus and the rise of Alistipes in OVA+Lamb and OVA+Fish group. At the same time, we use LEFSe analysis to find that Lachnospiraceae and Ruminococcaceae are the main changing factors in the OVA+Lamb group. Lachnospiraceae and Ruminococcaceae are the two richest families in Clostridia found in the mammalian intestinal environment, which were reported to degrade indigestible fibers to produce SCFA, thereby maintaining intestinal health. ${ }^{44}$ Lachnospiraceae can convert lactic acid or acetic acid into butyric acid under the action of $\mathrm{CoA}$, thus relieving visceral hypersensitivity. ${ }^{45}$ Nevertheless, higher abundance of Lachnospiraceae and Ruminococcaceae was also reported to drive a discrete intestinal microbial signature in patients of ankylosing spondylitis. ${ }^{46}$ What's more, some researchers have found that a long-term diet will divide the intestinal flora into three bacteria-dominated enterotypes, while Ruminococcus is one of the dominant bacteria. ${ }^{47}$ The long-term consumption of lamb and fish to mice may lead to the change of enterotype, which probably tends to impact intestinal homeostasis negatively. Therefore, whether these two genera benefit or do harm to human health needs more evidences to explore.

Finally, both ILC2 and Treg play an essential role in maintaining the homeostasis of the intestinal flora. We found that lambs affect the differentiation of Treg in the lungs and ILC2 in the small intestine, while fish can affect the differentiation of Treg in the small intestine. ILC2 is the primary producer of IL-10 in the gut, ${ }^{48}$ and Alistipes mentioned above can obviously proliferate in IL-10 mediated enteritis. ${ }^{42}$ In OVA+Lamb groups, both intestinal ILC2 and Alistipes increased, suggesting that they may have synergies in the intestinal burden. ILC2 can inhibit Treg activation through ICOS/ICOS ligand, which contributes to airway hyperresponsiveness. ${ }^{49}$ In our study, the lung ILC2 of the model group increased significantly, and the Treg decreased significantly, which may be the result of the interaction between ILC2 and Treg.

In brief, our study indicates that long-term diet of lamb meat or Basa fish can aggravate pulmonary inflammation and shape the gut microbial communities in asthmatic mice, especially lamb meat. Besides, ILC2 and Treg probably contribute to this exacerbation. How gut microbiota crosstalk with these ILC2 and Treg in asthmatic mice needs to be further investigated.

\section{Funding}

This research was funded by National Natural Science Foundation of China (No.81473656) and Fundamental Research Funds for Central Universities (No.2018JYBZZ-JS008 and No.2019-JYB-TD014).

\section{Disclosure}

All the authors declare no conflicts of interest in this work.

\section{References}

1. World Health Organization. The global asthma report. Available from: http://www.globalasthmareport.org. Accessed September 23, 2020.

2. Bateman ED, Hurd SS, Barnes PJ, et al. Global strategy for asthma management and prevention: GINA executive summary. Eur Respir J. 2008;31(1):143-178. doi:10.1183/09031936.00138707

3. Willett W. Diet and health: what should we eat. Science. 1994;264 (5158):532-537. doi:10.1126/science.8160011

4. Guilleminault L, Williams EJ, Scott HA, Berthon BS, Jensen M, Wood LG. Diet and asthma: is it time to adapt our message. Nutrients. 2017;9(11):1227. doi:10.3390/nu9111227

5. Deversux G, Seaton A. Diet as a risk factor for atopy and asthma. $J$ Allergy Clin Immunol. 2005;115(6):1109-1117. doi:10.1016/j. jaci.2004.12.1139

6. Huang D. A literature study on "stimulating foods" in Chinese medicine dietary taboos. [dissertation]. China: Beijing University of Chinese Medicine; 2009. 
7. Zhu F, Xie G. Discussion on Chinese medicine diet taboo-fawu. Chin $J$ Tradit Chin Med Pharm. 2018;7.

8. Hill CS DJ, Hosking FM, De Benedictis AP, et al. Confirmation of the association between high levels of immunoglobulin E food sensitization and eczema in infancy: an international study. Clin Exp Allergy. 2008;8(1):61-68. doi:10.1111/j.1365-2222.2007.02861.x

9. Mckenzie C, Tan J, Macia L, Mackay CR. The nutrition-gut microbiome-physiology axis and allergic diseases. Immunol Rev. 2017;278(1):277-295. doi:10.1111/imr.12556

10. Hecke TV, Vrieze JD, Boon N, Vos WHK, Vossen E, Smet SD. Combined consumption of beef-based cooked mince and sucrose stimulates oxidative stress, cardiac hypertrophy, and colonic outgrowth of desulfovibrionaceae in rats. Mol Nutr Food Res. 2019;63 (2):e1800962. doi:10.1002/mnfr.201800962

11. Ge Y, Lin S, Li B, et al. Oxidized pork induces oxidative stress and inflammation by altering gut microbiota in mice. Mol Nutr Food Res. 2020;64(2):e1901012. doi:10.1002/mnfr.201901012

12. Li N, Wu X, Zhuang W, et al. Fish consumption and multiple health outcomes: umbrella review. Trends Food Sci Technol. 2020;99:273-283. doi:10.1016/j.tifs.2020.02.033

13. Earl CS, An S, Ryan RP. The changing face of asthma and its relation with microbes. Trends Microbiol. 2015;23(7):408-418. doi:10.1016/j. tim.2015.03.005

14. Abrahamsson TR, Jakobsson HE, Andersson AF, Björkstén B, Engstrand L, Jenmalm MC. Low gut microbiota diversity in early infancy precedes asthma at school age. Clin Exp Allergy. 2014;44 (6):842-850. doi:10.1111/cea.12253

15. Russell SL, Gold MJ, Willing BP, Thorson L, McNagny KM, Finlay BB. Perinatal antibiotic treatment affects murine microbiota, immune responses and allergic asthma. Gut Microbes. 2013;4 (2):158-164. doi:10.4161/gmic.23567

16. Akay HK, Bahar Tokman H, Hatipoglu N, et al. The relationship between Bifidobacteria and allergic asthma and/or allergic dermatitis: A prospective study of $0-3$ years-old children in Turkey. Anaerobe. 2014;28:98-103. doi:10.1016/j.anaerobe.2014.05.006

17. Wang W, Luo X, Zhang Q, He X, Zhang Z, Wang X. Bifidobacterium infantis relieves allergic asthma in mice by regulating Th1/Th2. Med Sci Monit. 2020;26:e920583-1-e920583-11. doi:10.12659/ MSM.920583

18. Wu CT, Chen PJ, Lee YT, Ko JL, Lue KH. Effects of immunomodulatory supplementation with Lactobacillus rhamnosus on airway inflammation in a mouse asthma model. J Microbiol Immunol Infect. 2014;49(5):625-635. doi:10.1016/j.jmii.2014.08.001

19. Lloyd CM. Regulatory $T$ cells in asthma. Immunity. 2009;31 (3):438-449. doi:10.1016/j.immuni.2009.08.007

20. Bettelli E, Carrier Y, Gao W, Korn T, Strom TB, Oukka M. Reciprocal developmental pathways for the generation of pathogenic effector TH 17 and regulatory T cells. Nature. 2006;441 (7090):235-238. doi:10.1038/nature04753

21. Morita H, Moro K, Koyasu S. Innate lymphoid cells in allergic and nonallergic inflammation. $J$ Allergy Clin Immunol. 2016;138 (5):1253-1264. doi:10.1016/j.jaci.2016.09.011

22. Wolterink RGJK, Kleinjan A, Nimwegen M, Bergen I, Bruijn M, Levani Y. Pulmonary innate lymphoid cells are major producers of IL-5 and IL-13 in murine models of allergic asthma. Eur J Immunol. 2012;42(5):1106-1116. doi:10.1002/eji.201142018

23. Haung Y, Guo L, Qiu J, et al. IL-25-responsive, lineage-negative KLRG1hi cells are multipotential 'inflammatory' type 2 innate lymphoid cells. Nature. 2015;16(2):161-169. doi:10.1038/ni.3078

24. Huang Y, Mao K, Chen X, et al. S1P-dependent interorgan trafficking of group 2 innate lymphoid cells supports host defense. Science. 2018;359(6371):114-119. doi:10.1126/science.aam5809

25. Mao K, Baptista AP, Tamoutounour S, et al. Innate and adaptive lymphocytes sequentially shape the gut microbiota and lipid metabolism. Nature. 2018;554(7691):255-259. doi:10.1038/ nature 25437
26. Ding $\mathrm{Y}, \mathrm{Xu}$ J, Bromberg JS. Regulatory $\mathrm{T}$ cell migration during an immune response. Trends Immunol. 2012;33(4):174-180. doi:10.1016/j.it.2012.01.002

27. Atarashi K, Honda K. Microbiota in autoimmunity and tolerance. Curr Opin Immunol. 2011;23(6):761-768. doi:10.1016/j. coi.2011.11.002

28. Hu Y, Li T, Chen X, Zhang H, Yu A, Wang G. Effects of stimulating food on allergic contact dermatitis in BALB/c mice. J Nanjing Univ Tradit Chin Med. 2013;6.

29. Xu W, Hu M, Zhang Q, Yu J, Su W. Effects of anthraquinones from Cassia occidentalis L. on ovalbumin-induced airways inflammation in a mouse model of allergic asthma. $J$ Ethnopharmacol. 2018;221:1-9. doi:10.1016/j.jep.2018.04.012

30. Li Q, Li DL, Zhang X, Zhang W, Zheng M. The E3 ligase VHL promotes group 2 innate lymphoid cell maturation and function via inhibition of glycolysis and induction of interleukin 33 receptor. Immunity. 2018;48(2):258-270. doi:110.1016/j.immuni.2017.12.013

31. Andrianasolo RM, Hercberg S, Touvier M, et al. Association between processed meat intake and asthma symptoms in the french nutrinetsanté cohort. Eur J Nutr. 2020;59(4):1153-1162. doi:10.1007/ s00394-019-02011-7

32. Takemura Y, Sakurai Y, Honjo S, et al. The relationship between fish intake and the prevalence of asthma: the Tokorozawa childhood asthma and pollinosis study. Prev Med. 2002;34(2):221-225. doi:10.1006/pmed.2001.0978

33. US department of agriculture. FoodData central. Available from: https://fdc.nal.usda.gov/. Accessed September 23, 2020.

34. Antonio Ortenzi PP, Vincenzetti S, Beghelli D. Dietary properties of lamb meat and human health. Mediterr J Nutr Metab. 2011;4 (1):53-56. doi:10.1007/s12349-010-0032-9

35. Nagel G, Linseisen J. Dietary intake of fatty acids, antioxidants and selected food groups and asthma in adults. Eur J Nutr. 2005;59 (1):8-15. doi:10.1038/sj.ejcn.1602025

36. Kim EK, Ju SY. Asthma and dietary intake of fish, seaweeds, and fatty acids in korean adults. Nutrients. 2019;11(9):2187. doi:10.3390/ nu11092187

37. Broadfield EC, McKeever TM, Whitehurst A, et al. A case-control study of dietary and erythrocyte membrane fatty acids in asthma. Clin Exp Allergy. 2004;34(8):1232-1236. doi:10.1111/j.13652222.2004.02032.x

38. Cani PD, Amar J, Iglesias MA, et al. Metabolic endotoxemia initiates obesity and insulin resistance. Diabetes. 2007;56(7):1761-1772. doi: $10.2337 / \mathrm{db} 06-1491$

39. Fritsche KL. The science of fatty acids and inflammation. Adv Nutr. 2015;6(3):293S301S. doi:10.3945/an.114.006940

40. Mueller K, Ash C, Pennisi E, Smith O. The gut microbiota. Science. 2012;336(6086):1245. doi:10.1126/science.336.6086.1245

41. Legatzki A, Rösler B, Mutius EV. Microbiome diversity and asthma and allergy risk. Curr Allergy Asthma Rep. 2014;14(10):466. doi:10.1007/s11882-014-0466-0

42. Gerner RR, Moschen AR, Wang J, et al. Lipocalin2 deficiency results in colitis exacerbation and tumor development in IL-10 mice by Alistipes overgrowth. Z Gastroenterol. 2016;54(05). doi:10.1055/ s-0036-1583976

43. Yan F, Li N, Shi J, et al. Lactobacillus acidophilus alleviates type 2 diabetes by regulating hepatic glucose and lipid metabolism and gut microbiota in mice. Food Funct. 2019;10(9):5804-5815. doi:10.1039/c9fo01062a

44. Biddle A, Stewart LC, Susan Leschine JB. Untangling the genetic basis of fibrolytic specialization by lachnospiraceae and ruminococcaceae in diverse gut communities. Diversity. 2013;5(3):627-640. doi:10.3390/d5030627

45. Zhang J, Song L, Wang Y, et al. Beneficial effect of butyrate-producing Lachnospiraceae on stress-induced visceral hypersensitivity in rats. $J$ Gastroenterol Hepatol. 2019;34 (8):1368-1376. doi:10.1111/jgh.14536 
46. Costello ME, Ciccia F, Willner D, et al. Brief report: intestinal dysbiosis in ankylosing spondylitis. Arthritis Rheumatol. 2015;67 (3):686-691. doi:10.1002/art.38967

47. Wu G, Chen J, Hoffmann C, et al. Linking long-term dietary patterns with gut microbial enterotypes. Science. 2011;334(6052):105-108. doi: $10.1126 /$ science

48. Bando JK, Gilfillan S, Di Luccia B, et al. ILC2s are the predominant source of intestinal ILC-derived IL-10. J Exp Med. 2020;217(2): e20191520. doi:10.1084/jem.20191520
49. Rigas D, Lewis G, Aron JL, et al. Type 2 innate lymphoid cell suppression by regulatory $\mathrm{T}$ cells attenuates airway hyperreactivity and requires inducible T-cell costimulator-inducible T-cell costimulator ligand interaction. $J$ Allergy Clin Immunol. 2017;139 (5):1468-1477. doi:10.1016/j.jaci.2016.08.034

\section{Publish your work in this journal}

The Journal of Asthma and Allergy is an international, peer-reviewed open-access journal publishing original research, reports, editorials and commentaries on the following topics: Asthma; Pulmonary physiology; Asthma related clinical health; Clinical immunology and the immunological basis of disease; Pharmacological interventions and new therapies. The manuscript management system is completely online and includes a very quick and fair peer-review system, which is all easy to use. Visit http://www.dovepress.com/testimonials.php to read real quotes from published authors. 